Case Report

\title{
Cytological Features of a Variant NUT Midline Carcinoma of the Lung Harboring the NSD3-NUT Fusion Gene: A Case Report and Literature Review
}

\author{
Shiho Kuroda, ${ }^{1}$ Shioto Suzuki, ${ }^{1}$ Akira Kurita, ${ }^{1}$ Mari Muraki, ${ }^{1}$ Yoichiro Aoshima, ${ }^{2}$ \\ Fumihiko Tanioka, ${ }^{1}$ and Haruhiko Sugimura ${ }^{3}$ \\ ${ }^{1}$ Division of Pathology, Iwata City Hospital, 512-3 Ookubo, Iwata, Shizuoka 438-8550, Japan \\ ${ }^{2}$ Division of Respiratory Medicine, Iwata City Hospital, 512-3 Ookubo, Iwata, Shizuoka 438-8550, Japan \\ ${ }^{3}$ Department of Tumor Pathology, Hamamatsu University School of Medicine, 1-20-1 Handayama, Higashi-ku, \\ Hamamatsu, Shizuoka 431-3192, Japan \\ Correspondence should be addressed to Shioto Suzuki; shiosuzuki-path@umin.net
}

Received 21 November 2014; Accepted 29 December 2014

Academic Editor: Imtiaz A. Chaudhry

Copyright (c) 2015 Shiho Kuroda et al. This is an open access article distributed under the Creative Commons Attribution License, which permits unrestricted use, distribution, and reproduction in any medium, provided the original work is properly cited.

Background. Nuclear protein in testis (NUT) midline carcinoma (NMC) is a very rare and aggressive malignancy. In more than two-thirds of these NMC cases, a fusion between NUT and BRD4 or BRD3 has been documented; other variants are rare. The cytology of NMC itself has been sparsely documented and that of variant NMC has never been reported. Case Presentation. A 36-year-old woman was admitted because of a rapidly progressing lung tumor with metastases to the breast and bone. We recently reported this patient as the first case of a variant NMC of the lung harboring an NSD3-NUT fusion, based on immunohistochemical and genetic analyses. Cytological material was available for the present review. A highly cellular smear contained a predominantly noncohesive pattern of monomorphic cells with diameters 2-2.5 times greater than those of small lymphocytes, with a roundto-oval nucleus, slightly irregular nuclear contours, variably prominent nucleoli, scant cytoplasm, and identifiable mitotic figures. Foci of stratification and overt pearl formation, including a dyskeratocyte, were occasionally observed. The necrotic background contained naked nuclei, karyorrhectic debris, apoptotic cells, and macrophages phagocytizing karyorrhectic debris; nuclear crushing was noted. Conclusion. The cytological features of a variant NMC of the lung are described for the first time.

\section{Introduction}

Nuclear protein in testis (NUT) midline carcinoma (NMC) is a recently recognized entity that is characterized by undifferentiated morphological features and immunoreactivity to NUT [1]. This disease is a very rare [2-4] and aggressive $[3,4]$ malignancy that most often occurs in the midline of the body, including the head and neck and the mediastinum $[2,4]$. Currently, the diagnosis of NMC depends on the identification of a rearrangement involving the NUT locus at $15 \mathrm{q} 14$ that generates a specific fusion transcript with a member of the bromodomain-containing protein (BRD) family, such as BRD4 located on chromosome 19p13.1. In more than two-thirds of NMC cases, a gene fusion between NUT and $B R D 4$ or $B R D 3$ has been documented $[2,5-7]$; other variant fusions are rare [6]. Recently, we described a variant NMC in which an NSD3- (nuclear receptor binding SET domain 3) NUT rearrangement was identified in the primary tissue using $5^{\prime}$-rapid amplification of the cDNA end (RACE); the fusion was validated using fluorescence in situ hybridization (FISH) [8].

Little information is available on the cytological features of NMC. Three reports have described the cytological features of 4 common NMC cases $[9,10]$ and another NMC case in which the gene rearrangement was not analyzed [11], and no information on the cytology of variant NMC is presently 
available. We herein describe, for the first time, the cytological features of a variant NMC of the lung harboring an NSD3NUT fusion gene.

\section{Clinical Summary}

A 36-year-old woman sought medical advice because of a cough accompanied by wheezing with a 2-month duration. An enhanced computed tomography scan performed at the time of hospitalization revealed a mass $(75 \times 38 \times 35 \mathrm{~mm}$ in size) in the left lung that extended to the middle mediastinum; metastatic lesions in the liver, breast, bones, and lymph nodes were also detected [8]. A transbronchial biopsy (TBB) of the lung tumor and an endobronchial ultrasoundguided transbronchial needle aspiration (EBUS-TBNA) of a lower mediastinal lymph node with lung tumor involvement were performed.

\section{Materials and Methods}

The aspiration material obtained from the lymph node was separated into two parts; its small portion was available for cytological investigation. The specimen was air-dried and Hemacolor-stained (MERCK, Darmstadt, Germany) at bedside according to the manufacturer's instructions and a portion was fixed in 95\% ethanol and stained with Papanicolaou as ordinary methods. The larger part of the aspiration material obtained from the lymph node and the biopsy material taken from the lung tumor were immediately immersed in $20 \%$ buffered neutral formalin, fixed overnight, and embedded in paraffin. These specimens were then sectioned and used for hematoxylin-eosin staining, immunohistochemistry with antibodies for EMA (DAKO, Glostrup, Denmark), p63 (Santa Cruz Biotechnology, Dallas, TX, USA), cytokeratin AE1/AE3 (DAKO), cytokeratin CAM 5.2 (Becton, Dickinson and Company, CA, USA), CD138 (DAKO), vimentin (DAKO), and others, and FISH, as reported previously [8].

3.1. Cytological Findings. An overview of the Papanicolaoustained smear showed the specimen to be highly cellular with loosely cohesive cells and/or isolated cells (Figure 1). The cells were 2-2.5 times greater in diameter than that of a small lymphocyte. The nuclei were round to oval in shape and had slightly irregular contours, with one or more prominent nucleoli (Figure 1). The chromatin was hyperchromatic and finely granular in most of the cells or vesicular in occasional cells. The main cells, which had scant cytoplasm and an indistinct cell border, often formed loosely cohesive clusters (Figure 2). These clusters were in contact with foci of stratification (Figure 2), which consisted of occasional cells with a clear cell border and moderately delicate cytoplasm, often with cytoplasmic coarse vacuoles (Figure 3, arrowhead) that were negative for epithelial mucin. In addition, a few cells showed an overt pearl formation, including a dyskeratocyte (Figure 3, arrow), whereas occasional small apoptotic cells with orange G-colored cytoplasm were scattered throughout the specimen. A glandular structure was not observed.

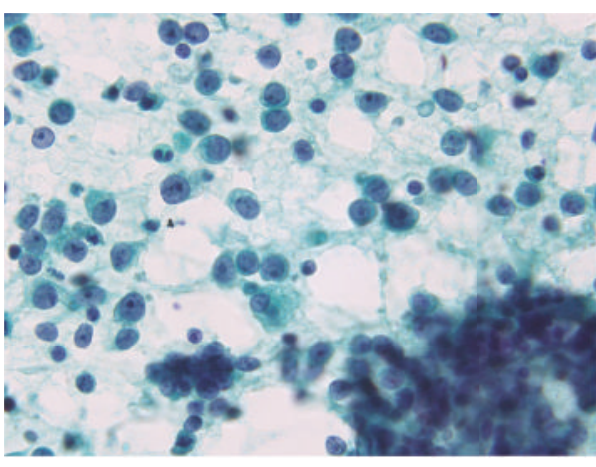

FIGURE 1: Papanicolaou-stained smear showing a high cellularity with loosely cohesive cells or isolated cells. The cells were 2-2.5 times greater in diameter than that of a small lymphocyte. The nuclei were round to oval in shape with slightly irregular contours and contained one or more prominent nucleoli. The chromatin was hyperchromatic and finely granular.

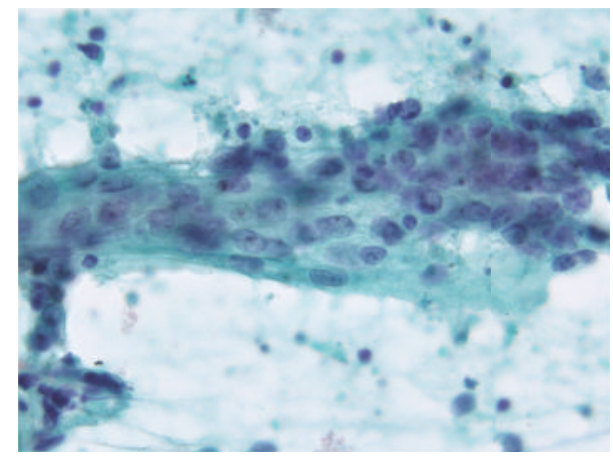

FIgURe 2: Papanicolaou-stained smear showing loosely cohesive clusters and stratification. The main cells exhibited scant cytoplasm and an indistinct cell border, forming loosely cohesive clusters (right side) that were in contact with foci of stratification (center-left side), which consisted of occasional cells with a clear cell border and moderately delicate cytoplasm.

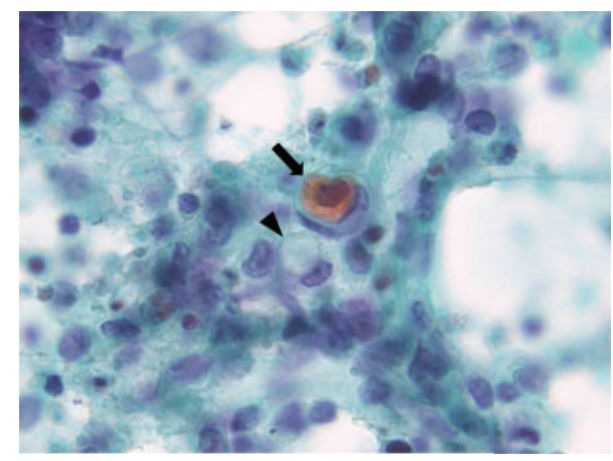

FIGURE 3: Papanicolaou-stained smear showing pearl formation. A few cells showed overt pearl formation, including a dyskeratocyte (arrow), implying keratinization. Occasional cells with moderately delicate cytoplasm and cytoplasmic coarse vacuoles (arrow head) are visible. 


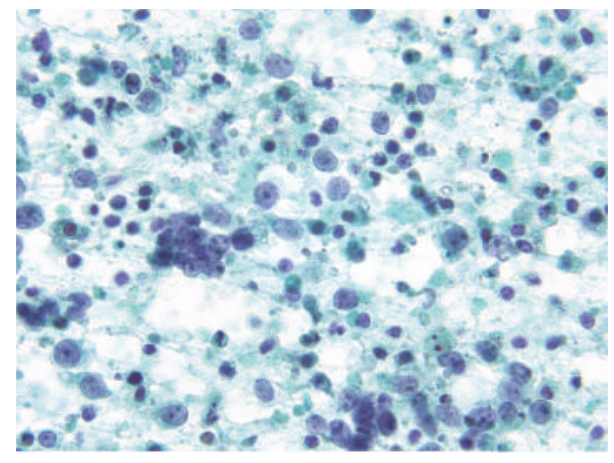

FIGURE 4: Papanicolaou-stained smear showing a necrotic background containing naked nuclei, karyorrhectic debris, apoptotic cells, and macrophages phagocytizing karyorrhectic debris.

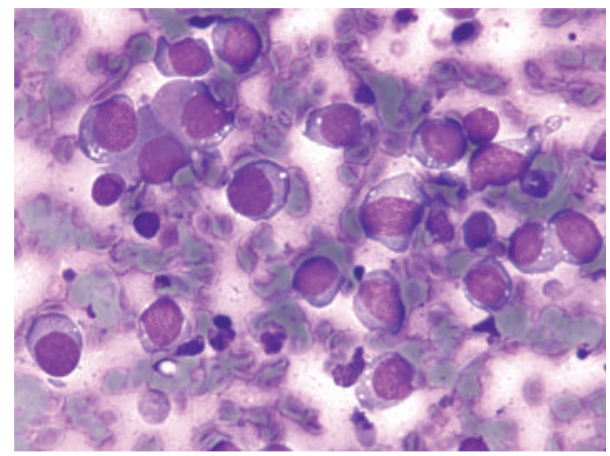

Figure 5: Hemacolor-stained smear showing cells bearing basophilic cytoplasm focally with several cytoplasmic fine vacuoles.

Pleomorphism of the cells was not prominent. Mitotic figures were often identified. The necrotic background contained naked nuclei, karyorrhectic debris, apoptotic cells, and macrophages phagocytizing karyorrhectic debris (Figure 4); nuclear crushing was noted.

On the other hand, the Hemacolor-stained smear showed occasional cells bearing delicate basophilic cytoplasm focally with several cytoplasmic fine vacuoles (Figure 5), whereas these cytoplasmic fine vacuoles were not identified in the Papanicolaou-stained smear material.

\subsection{Histological, Immunohistological, and FISH Findings.} Details of the histological and molecular features of this case have been previously reported [8]. Briefly, only a small amount of biopsy was available for histological investigation, and it revealed an undifferentiated neoplasm with necrosis (Figure 6). In this material, squamous differentiation, which is a possible characteristic of variant NMC, was not apparent.

Immunohistochemical staining demonstrated focal positivity for EMA, p63, cytokeratin AE1/AE3, cytokeratin CAM 5.2, CD138, and vimentin. In addition, a nuclear staining pattern for NUT was evident (Figure 7). Furthermore, FISH analyses revealed an NSD3-NUT rearrangement (Figure 8), whereas BRD3/4-NUT fusion genes were not identified.

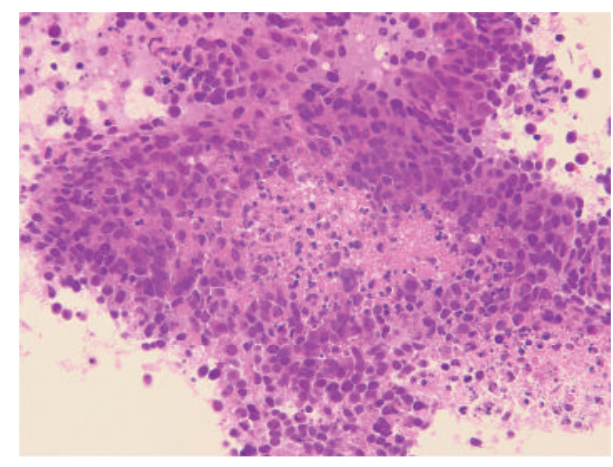

FIGURE 6: Representative histological image showing sheets of undifferentiated malignant cells with focal necrosis.

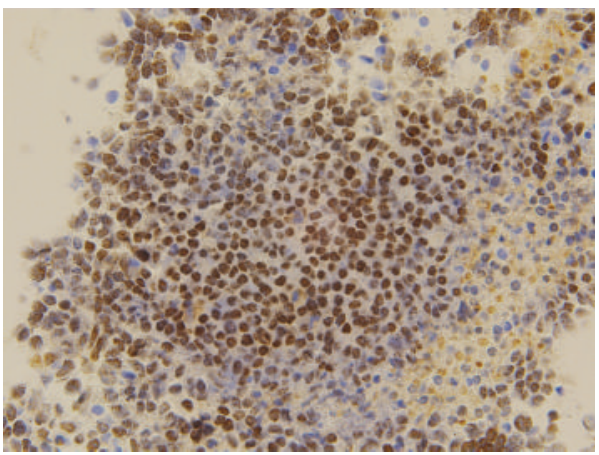

FIGURE 7: Immunohistochemistry showing a nuclear staining pattern for NUT.

3.3. Treatment and Follow-Up. The patient received chemoradiation therapy for 10 months after her diagnosis. However, the patient died with disease progression at 10 months after the diagnosis. Autopsy was not permitted.

\section{Discussion}

The histological features of NMCs range from entirely undifferentiated carcinomas to carcinomas with prominent squamous differentiation $[2,7,12-15]$. Thus, a diagnosis of NMC based solely on morphology can be difficult. Previous studies have described the cytological features of 4 common NMCs harboring a BRD3/4-NUT fusion gene $[9,10]$ and another NMC case in which the gene rearrangement was not analyzed [11]; the cytological characteristics of these NMCs showed a highly cellular, predominantly noncohesive pattern of relatively small cells with a round nucleus, scant cytoplasm, irregular nuclear contours, variably prominent nucleoli, and identifiable mitotic figures. These findings were also observed in the present variant NMC case. Thus, the previously reported findings imply that the cytological characteristics of NMC are nonspecific and similar to those of undifferentiated carcinoma.

Keratinization has not been identified in previous studies examining the cytology of NMCs [9-11]. In theory, overt keratinization is very important and sometimes pathognomonic for a scrutinized diagnosis of NMC; that is, it is assumed 


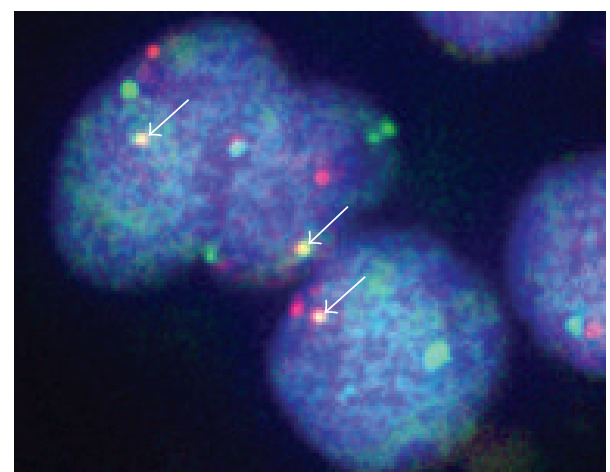

FIGURE 8: A dual-color FISH analysis showing the fusion gene as a single yellow (overlapping) signal (arrows), including a green (NSD3) and an orange (NUT) signal.

to be a relatively characteristic finding of NMC harboring a NUT gene rearrangement involving a gene other than $B R D 4$ [16]. Keratinization in surgical material, however, is notorious for evading observation because its distribution is often very focal $[6,16]$, and sampling biases often occur. The cytology of the present NMC variant showed overt pearl formation, including a dyskeratocyte, and the stratification in contact with loosely cohesive clusters; we believe that this finding corresponds to abrupt keratinization, which has been reported as a peculiar characteristic of variant NMC [16] but was not recognized in our corresponding histology specimen [8]. Thus, the combination of cytological and histological findings may help to reveal keratinization concurrent with stratification in undifferentiated carcinoma, which is one clue for a diagnosis of NMC, especially variant NMC, rather than other tumors.

The Hemacolor-stained smear showed occasional cells possessing delicate basophilic cytoplasm with several fine vacuoles, which were not observed in our Papanicolaoustained smear. In a previous NMC case harboring a BRD3NUT fusion gene, the cells were described as possessing delicate to finely vacuolated cytoplasm, although we could not observe the cytoplasm in detail because of the low magnification of the published figures [10]. On the other hand, these cytoplasmic fine vacuoles were not observed in NMC cases harboring a BRD4-NUT fusion gene [9]. Further studies are needed to clarify whether these cytoplasmic fine vacuoles are specific for NMC harboring a fusion gene involving NUT and a gene other than BRD4.

In conclusion, although the distinction of NMC from other poorly differentiated carcinomas based solely on morphology is difficult, cytological investigation is helpful, especially for identifying abrupt keratinization, which histopathological investigations can miss because of sampling biases. Our experience has shown that the identification of the following clues may suggest a diagnosis of NMC; overt pearl formation including a dyskeratocyte, stratification, and cytoplasmic fine vacuoles, especially in cases where the initial suspected diagnosis was "undifferentiated or poorly differentiated carcinoma with little pleomorphism." Furthermore, the identification of this entity is critical, and immunohistochemistry or FISH studies should be considered for the identification of NUT gene rearrangements.

\section{Conflict of Interests}

The authors have no conflicts of interest to declare.

\section{Acknowledgments}

The authors appreciate the technical assistance of all the clinical and technical staff members of the Pathology Division of Iwata City Hospital. This contribution was supported, in part, by Grants-in-Aid for the US-Japan Cooperative Medical Science Program; the National Cancer Center Research and Development Fund; a grant for priority areas from the Japanese Ministry of Education, Culture, Sports, Science and Technology (221S0001); and Grants-in-Aid for Cancer Research from the Japanese Ministry of Health, Labour and Welfare (23120201 and 10103838), the Smoking Research Foundation, and the Princess Takamatsu Cancer Research Fund.

\section{References}

[1] H. Haack, L. A. Johnson, C. J. Fry et al., "Diagnosis of NUT midline carcinoma using a NUT-specific monoclonal antibody," The American Journal of Surgical Pathology, vol. 33, no. 7, pp. 984-991, 2009.

[2] C. A. French, "NUT midline carcinoma," Cancer Genetics and Cytogenetics, vol. 203, no. 1, pp. 16-20, 2010.

[3] D. E. Bauer, C. M. Mitchell, K. M. Strait et al., "Clinicopathologic features and long-term outcomes of NUT midline carcinoma," Clinical Cancer Research, vol. 18, no. 20, pp. 5773-5779, 2012.

[4] E. B. Stelow, "A review of NUT midline carcinoma," Head and Neck Pathology, vol. 5, no. 1, pp. 31-35, 2011.

[5] C. A. French, I. Miyoshi, I. Kubonishi, H. E. Grier, A. R. PerezAtayde, and J. A. Fletcher, "BRD4-NUT fusion oncogene: a novel mechanism in aggressive carcinoma," Cancer Research, vol. 63, no. 2, pp. 304-307, 2003.

[6] C. A. French, "Pathogenesis of NUT midline carcinoma," Annual Review of Pathology: Mechanisms of Disease, vol. 7, pp. 247-265, 2012.

[7] S. Suzuki, N. Kurabe, H. Minato et al., "A rare Japanese case with a NUT midline carcinoma in the nasal cavity: a case report with immunohistochemical and genetic analyses," Pathology Research and Practice, vol. 210, no. 6, pp. 383-388, 2014.

[8] S. Suzuki, N. Kurabe, I. Ohnishi et al., "NSD3-NUT-expressing midline carcinoma of the lung: first characterization of primary cancer tissue," Pathology-Research and Practice. In press.

[9] A. M. Bellizzi, C. Bruzzi, C. A. French, and E. B. Stelow, "The cytologic features of NUT midline carcinoma," Cancer Cytopathology, vol. 117, no. 6, pp. 508-515, 2009.

[10] B. Zhu, W. Laskin, Y. Chen et al., "NUT midline carcinoma: a neoplasm with diagnostic challenges in cytology," Cytopathology, vol. 22, no. 6, pp. 414-417, 2011.

[11] H. S. Park, Y. S. Bae, S. O. Yoon et al., "Usefulness of nuclear protein in testis (NUT) immunohistochemistry in the cytodiagnosis of NUT midline carcinoma: a brief case report," The Korean Journal of Pathology, vol. 48, no. 4, pp. 335-338, 2014. 
[12] A. G. Evans, C. A. French, M. J. Cameron et al., "Pathologic characteristics of NUT midline carcinoma arising in the mediastinum," American Journal of Surgical Pathology, vol. 36, no. 8, pp. 1222-1227, 2012.

[13] J. A. Bishop and W. H. Westra, "NUT midline carcinomas of the sinonasal tract," American Journal of Surgical Pathology, vol. 36, no. 8, pp. 1216-1221, 2012.

[14] B. N. Davis, R. G. Karabakhtsian, A. L. Pettigrew, S. M. Arnold, C. A. French, and Y. M. Brill, "Nuclear protein in testis midline carcinomas: a lethal and underrecognized entity," Archives of Pathology and Laboratory Medicine, vol. 135, no. 11, pp. 14941498, 2011.

[15] E. B. Stelow, A. M. Bellizzi, K. Taneja et al., "NUT rearrangement in undifferentiated carcinomas of the upper aerodigestive tract," The American Journal of Surgical Pathology, vol. 32, no. 6, pp. 828-834, 2008.

[16] C. A. French, J. L. Kutok, W. C. Faquin et al., "Midline carcinoma of children and young adults with NUT rearrangement," Journal of Clinical Oncology, vol. 22, no. 20, pp. 4135-4139, 2004. 


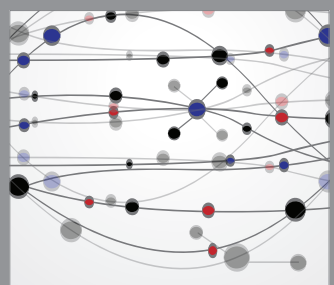

The Scientific World Journal
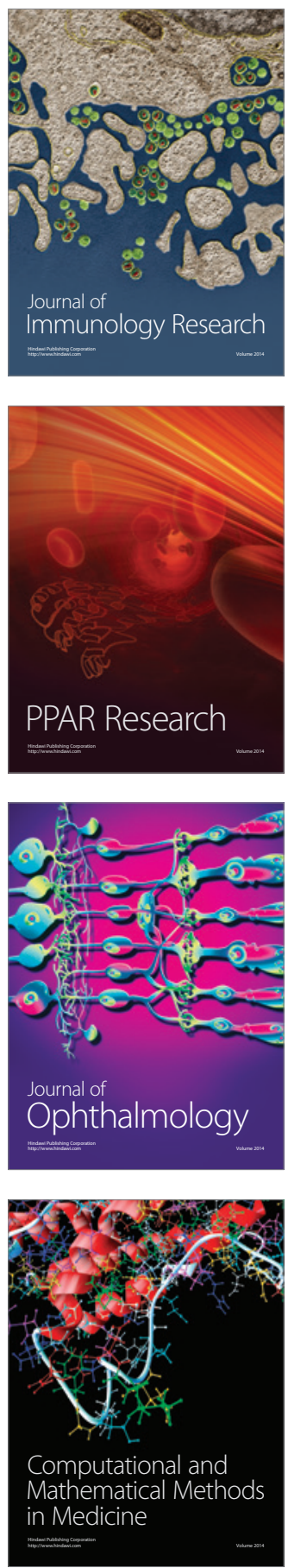

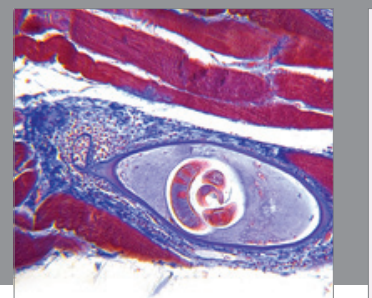

Gastroenterology

Research and Practice
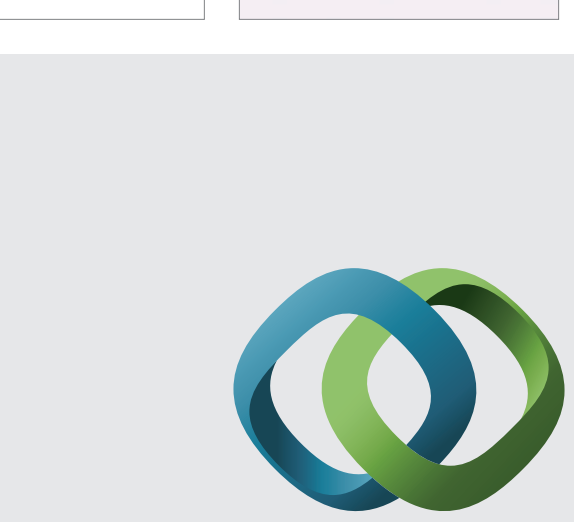

\section{Hindawi}

Submit your manuscripts at

http://www.hindawi.com
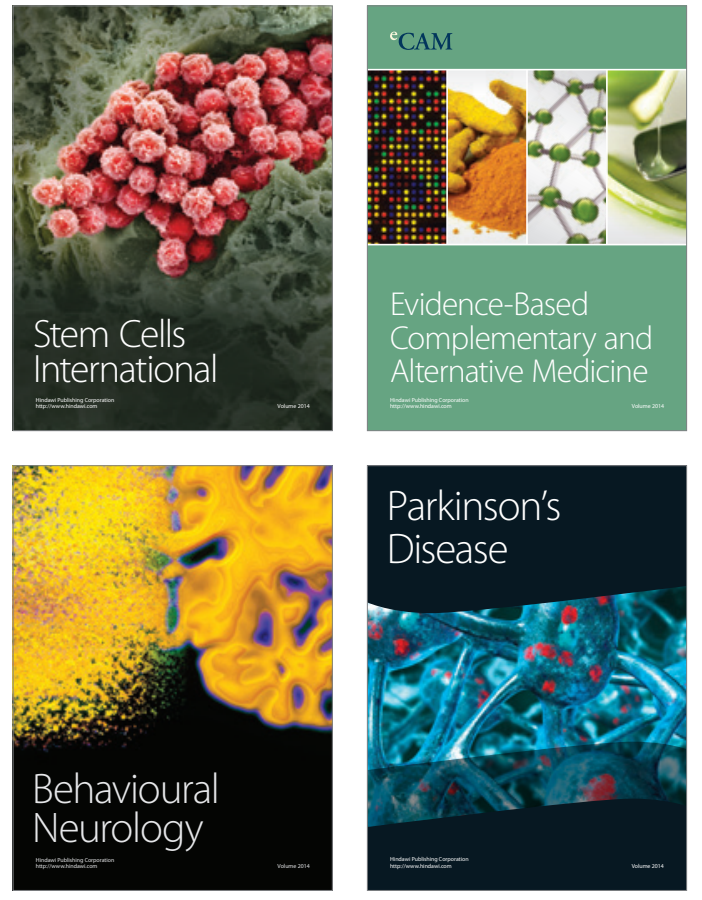
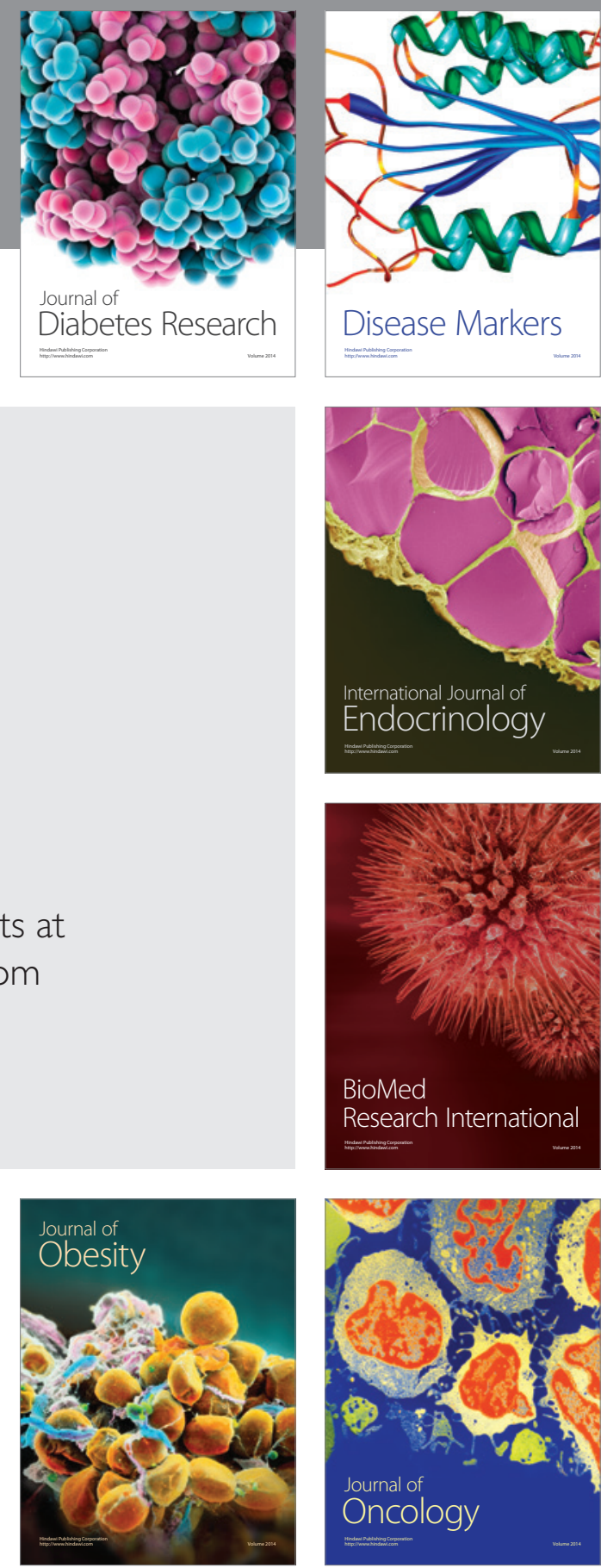

Disease Markers
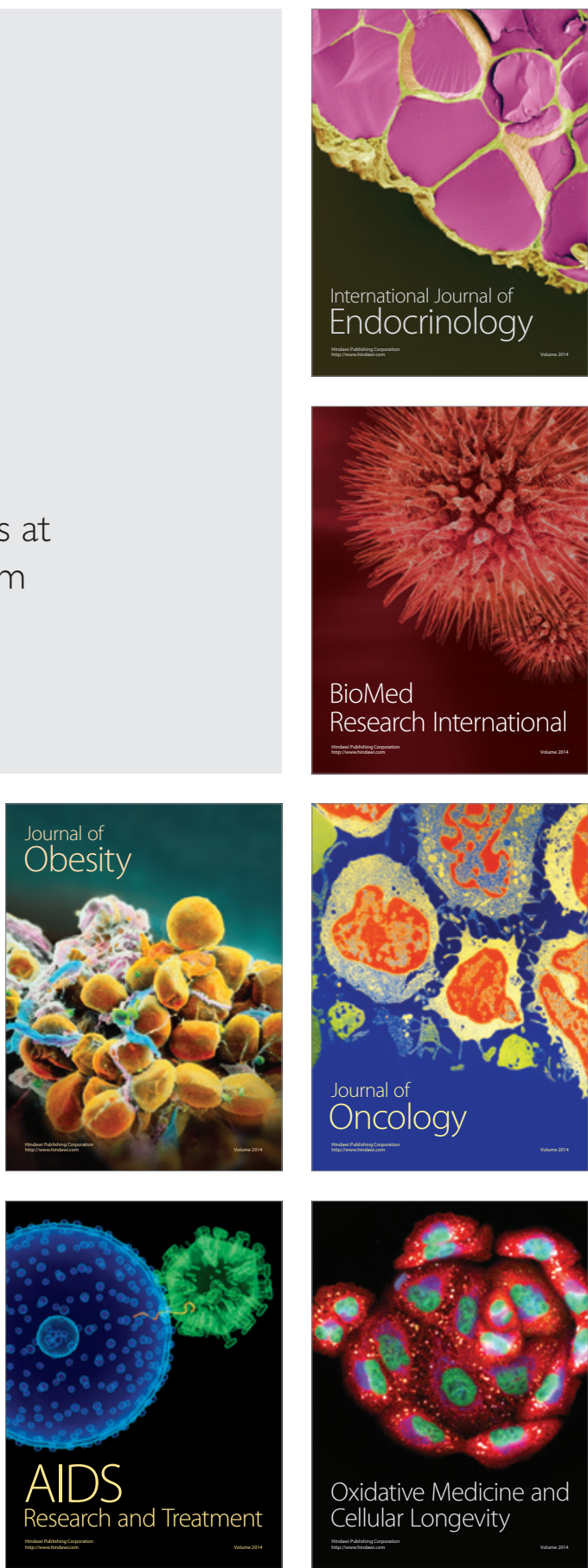